\title{
The asymmetrical influence of increasing time-on-task on attentional disengagement
}

RebeccaE. Paladini ${ }^{1}$, Lorenzo Diana ${ }^{1}$, Thomas Nyffeler ${ }^{1,2,3}$, Urs P. Mosimann ${ }^{1,4}$, Tobias Nef $^{1,5}$, René M. Müri ${ }^{1,3,6}$, Dario Cazzoli ${ }^{1,5}$

${ }^{1}$ Gerontechnology and Rehabilitation Group, University of Bern, Bern, Switzerland; ${ }^{2}$ Center of Neurology and Neurorehabilitation, LuzernerKantonsspital, Lucerne, Switzerland; ${ }^{3}$ Perception and Eye Movement Laboratory, Departments of Neurology and Clinical Research, University Hospital Inselspital, University of Bern, Bern, Switzerland; ${ }^{4}$ University Hospital of Old Age Psychiatry, University of Bern, Bern, Switzerland; ${ }^{5}$ ARTORG Center for Biomedical Engineering Research, University of Bern, Bern, Switzerland; ${ }^{6}$ Center for Cognition, Learning and Memory, University of Bern, Bern, Switzerland

Corresponding author:

Dario Cazzoli, PhD

Gerontechnology\& Rehabilitation Group

ARTORG Center for Biomedical Engineering Research

Murtenstrasse 50

3008 Bern

Switzerland

Tel. +41316324697

Fax. +41316327576

dario.cazzoli@artorg.unibe.ch 


\section{Abstract}

Increasing time-on-task leads to fatigue and, as shown by previous research, differentially affects the deployment of visual attention towards the left and the right visual space. In healthy participants, an increasing rightward bias is commonly observed with increasing time-on-task. Yet, it is unclear whether specific mechanisms involved in the spatial deployment of visual attention are differentially affected by increasing time-on-task.

The aim of the present study was to investigate whether prolonged time-on-task would affect a specific mechanism of visuo-spatial attentional deployment, namely attentional disengagement, in an asymmetrical fashion. For this purpose, we administered to healthy participants a prolonged gap/overlap saccadic paradigm, with left- and right-sided target stimuli. This oculomotor paradigm allowed to quantify disengagement costs according to the direction of the subsequent attentional shifts, and to evaluate the temporal development of disengagement costs with increasing time-ontask.

Our results show that, with increasing time-on-task, participants demonstrated significantly lower disengagement costs for rightward compared to leftward saccades. These effects were specific, since concurring side differences of saccadic latencies were found for overlap trials (requiring attentional disengagement), but not for gap trials (requiring no or less attentional disengagement). Moreover, the results were paralleled by a non-lateralised decrease in saccadic peak velocity with increasing time-on-task, a common finding indicating an increasing level of fatigue.

Ourfindings support the ideathat non-spatial attentional aspects, such as fatigue due to increasing time-on-task, can have a substantialinfluence on the spatial deployment of visual attention, in particular on its disengagement, depending on the direction of the subsequent attentional shift.

Keywords: Spatial attention; non-spatial attention; fatigue; eyemovements. 


\section{Introduction}

When confronted with prolonged cognitive tasks, participants gradually develop fatigue, which can be generally defined as a state of weariness and drowsiness, reducing efficiency and the desire for mental effort (Grandjean, 1979; Lal \& Craig, 2001; Pereverzev, 2014). Fatigue due to increasing timeon-task has been shown to influence the deployment of visual attention in space, thereby having asymmetrical effects, i.e., unequally influencing attentional deployment towards the left and the right visual space. With increasing time-on-task, healthy participants show an increasing rightward bias (e.g., Benwell, Harvey, Gardner, \&Thut, 2013; Manly, Dobler, Dodds, \& George, 2005; Newman, O'Connell, \&Bellgrove, 2013). This phenomenon is referred to as the "time-on-task effect" (Dufour, Touzalin, \&Candas,2007; Manly et al., 2005).

However, to date, it is not clear whether fatigue due to increasing time-on-task differentially affects the multiple mechanisms involved in the spatial deployment of visual attention. The influential theoretical framework proposed by Posner and colleagues (Posner, Cohen, \&Rafal, 1982; Posner \& Petersen, 1990) conceptualises attentional deployment in space as a function of three distinct processes. First, attention has to be disengaged from the current object of fixation, second, it has to be shifted to a target stimulus, and, third, it has to be re-engaged on the target. Among these processes, some previous results suggest that attentional disengagement, in particular, may be prone to the effects of fatigue (see Bocca\& Denise, 2006 for the effects of fatigue due to sleep deprivation on attentional disengagement). However, it is not known whether the effects of fatigue on attentional disengagement are compatible with the aforementioned "time-on-task effect", namely: a) whether the effects are observable with increasing time-on-task; and, b) more crucially, whether, the effects are asymmetrical, i.e., unequally affect attentional deployment towards the left and the right side of space.

An efficient method to quantify attentional disengagement is to measure saccadic eye movements during different tasks. The cortical representation of eye movements strongly overlaps with the one of visual attention (e.g., Corbetta et al., 1998; Nobre,Gitelman, Dias, \&Mesulam, 2000), and gaze and 
attention typically shift together in space when the eyes are free to move (e.g., Hoffman \&Subramaniam, 1995; Shepherd, Findlay, \& Hockey, 1986). Attentional disengagement can be explicitly quantified by contrasting the latencies of gap and overlap saccades. In a gap/overlap paradigm, participants are asked to fixate a central dot, and to then shift their gaze on a lateral target stimulus as quickly and as accurately as possible upon its appearance. Whilst the central fixation remains visible throughout overlap trials, it extinguishes prior to the onset of the target stimulus in gap trials. Generally, saccade latencies are shorter for gap compared to overlap trials (e.g., Saslow, 1967). This phenomenon has sometimes been termed the "gap effect", and is thought to reflect attentional disengagement. In fact, attention has to first be disengaged from the central fixation in overlap trials, whereas this process is not necessary in gap trials, due to the extinction of the central fixation (Fischer \&Breitmeyer, 1987; Fischer \& Weber, 1993; Jin\& Reeves, 2009). Hence, differences in saccadic latencies between overlap and gap trials reflect attentional "disengagement costs". Moreover, by manipulating the visual field (left or right) in which the saccade target is presented, potential asymmetrical effects on disengagement costs (i.e., depending on the direction of the next attentional shift) can be specifically assessed.

The aim of the present study was thus to investigate whether prolonged time-on-task, leading to increasing fatigue, would have an impact on attentional disengagement costs in healthy individuals, and, more crucially, whether attentional disengagement costs would be affected asymmetrically. For this purpose, we administered a gap/overlap saccadic paradigm, in which target stimuli were presented both in the left and in the right visual field, for a prolonged period of time. This approach allowed us to quantify the development of attentional disengagement costs with increasing time-ontask, and according to the direction of the subsequent attentional shifts. 


\section{Methods}

\subsection{Participants}

A total of 30 healthy subjects, with a mean age of 28.7 years $(S D=5.7$, range $=20-47$ ), participated in the study. 19 were female and 25 were right-handed, as assessed by the Edinburgh Handedness Inventory (Oldfield, 1970).

All participants had normal or corrected-to-normal visual acuity and gave written informed consent prior to the beginning of the study. The study was approved by the Ethics Committee of the State of Bern, and was conducted in compliance with the latest version of the Declaration of Helsinki.

\subsection{Stimuli and Materials}

Stimuli of the gap/overlap task consisted of light grey dots, subtending $.5^{\circ}$ visual angle, presented on a black background.A first grey dot was presented at the centre of the screen (central fixation), and a second greydot (target stimulus) was presented either to the left or to the right of the first one, on the horizontal midline, at an eccentricity of $12^{\circ}$ visual angle. Each trial of the gap/overlap task started with the presentation of the central fixation for 1200 or $1500 \mathrm{~ms}$ (random assignment). In the gap trials, the central fixation was extinguished for $200 \mathrm{~ms}$, after which the target stimulus was presented for $600 \mathrm{~ms}$ on the right or the left side. In the overlap trials, the central fixation remained on while the lateral target stimulus was additionally presented for $600 \mathrm{~ms}$. Both gap and overlap trials were then followed by a black screen for $1700 \mathrm{~ms}$ (inter-trial interval). A schematic depiction of a gap and an overlap trial is presented in Fig. 1.

Fig. 1 about here 
The experiment included 360 trials overall, organized in 30 blocks. Each block contained 12 trials: 3 gap and 3 overlap trials, repeated twice for left- and right-sided targets, respectively, in randomized order. The 360 trials were further divided into time intervals, whereby 120 trials (i.e., 10 blocks) made up one time interval, and each time interval was completed within approximately 8 minutes.

The participants' subjective level of fatigue was assessed by means of a visual analogue scale (VAS). Participants were asked to indicate their current level of fatigue by drawing a vertical mark on a 100 $\mathrm{mm}$ horizontal line, ranging from "I'm extremely fatigued, exhausted" (left extreme of the line) to "I'm not fatigued at all" (right extreme of the line).

\subsection{Apparatus}

Stimuli were presented on a 20-inch computer screen (Dell UltraSharp, Dell Inc., Round Rock, TX), with a resolution of $1600 \times 1200$ pixels, a refresh rate of $60 \mathrm{~Hz}$, and 16-bit colour depth. Stimulus presentation was performed by means of E-prime software (Psychology Software Tools, Inc., Sharpsburg, PA).

Participants' eye movements were recorded using an infrared, video-based eye-tracking system (Iview X Hi-Speed 2.0, Sensomotoric Instruments $\mathrm{GmbH}$, Teltow, Germany), with a sampling rate of $240 \mathrm{~Hz}$, a spatial resolution of typically $<.01^{\circ}$, and a gaze position accuracy of typically $.25^{\circ}-.5^{\circ}$ (largely dependent on calibration accuracy). The chin- and forehead-rest integrated in the system column ensured a constant viewing distance of $71.5 \mathrm{~cm}$.

\subsection{Procedure}

The experiment was conducted in a dimly lit room. Participants were instructed to fixate the central fixation dot, and to look at the lateral target stimulus as quickly and as accurately as possible upon its appearance. Participants were asked to refrain from blinking during the trails, and were encouraged 
to blink freely during the inter-trial-intervals. All participants completed 5 practice trials of the gap/overlap task.

Participants then performed 240 trials (i.e., 2 time intervals) of the gap/overlap task, after which they were allowed a break of 10 minutes. The break allowedto recalibrate the eye-tracking system, and allowed participantsto rate their fatigue level through the VAS. The break was followed by further 120 trials (i.e., 1 time interval) of the gap/overlap task. Participants were asked to rate, through the VAS, their subjective level of fatigue 4 times during the course of the experiment: before time interval 1, after time interval 2, before time interval 3, and after time interval 3.To further investigate the time course of performance on an even larger number of trials (and thus on a longer time-ontask), the experiment was extended by 360 additional trials in 10 of the 30 subjects. Thus, these subjects performed a total of 720 trials, with a 10-minute break after time intervals 2 and 4 . They rated, through the VAS, their subjective level of fatigue6 times during the course of the experiment: before time intervals 1, 3, and 5, and after time intervals 2, 4 and 6 (see Fig. 2 for a schematic representation of the timeline of the study).

Fig. 2 about here 


\subsection{Data Analysis}

Eye movement data were visually inspected in order to exclude trials containing errors, i.e., anticipatory saccades (saccade latencies<80 ms; Kingstone \& Klein, 1993), multiple-step saccades, saccades towards the opposite direction with respect to the target, and eye blinks. This procedure led to the exclusion of $5.28 \%$ of the trials. The number of errors did not change significantly over the time intervals, as indicated by separate repeatedmeasures analyses of variance (ANOVAs) for the first 3 time intervals $\left(N=30 ; F_{2,56}=0.442 ; p=0.645\right)$, and for all of the 6 time intervals $\left(N=10 ; F_{5,45}=\right.$ 1.84; $p=0.124)$. The following saccade parameters were then analysed: (1) saccade latency, i.e., the time interval between the appearance of the target stimulus and saccade initiation, in ms; (2) peak saccadic velocity, in $\%$; and,(3) saccade accuracy as reflected in the gain, i.e., the ratio between the required saccade amplitude (the distance between the central fixation and the target, $12^{\circ}$ ) and the actual amplitude of the produced saccade. For every parameter, median values were calculated for every participant, time interval, saccade direction, and task (i.e., gap or overlap). As the principal aim of this study consisted in investigating time-on-task effects on attentional disengagement, disengagement costs were explicitly quantifiedby subtracting saccade latencies in the gap trials from saccade latencies in the overlap trials. Disengagement costs have been shown to reflect the disengagement of attention (e.g., Fischer \&Breitmeyer, 1987). Calculating disengagement costs separately for left- and rightward saccades allowed to quantify attentional disengagement in relation to the direction of the subsequent attentional shift.

Separate analyses of the eye movement parameters (mean disengagement costs, mean saccadic latency, mean peak velocity, mean gain) were performed for the first 3 time intervals, which were completed by 30 subjects. Repeated measures ANOVAs with the within-subjects factors 'time interval' (levels: 1, 2, 3), 'saccade direction' (levels: leftward, rightward), and 'task' (levels: gap, overlap) were computed. Subsequently, in order to investigate the time course of performance on an even larger number of trials (and thus on a longer time-on-task), the data of the 10 subjects who had completed 6 time intervals were analysed separately. Repeated measures ANOVAs with the within- 
subjects factors 'time interval' (levels: 1, 2, 3, 4, 5, 6), 'saccade direction' (levels: leftward, rightward), and 'task' (levels: gap, overlap) were calculated.

The subjective level of fatigue, reported through the VAS by the participants, was assessed by measuring the distance between the right extreme of the scale ("I'm not fatigued at all") and the participants' mark, in mm. Hence, high scores indicate high levels of fatigue, and low scores indicate low levels of fatigue. The scores of the VAS were analysed separately for the 20 participants for whom the experiment ended after the completion of 3 time intervals, and for the 10 participants for whom the experiment continued for further 3 time intervals. A repeated measures (ANOVA) with the within-subjects factor 'time' (levels: before time interval 1, after time interval 2, before time interval 3, after time interval 3) was calculated on the VAS scores of the participants who completed a total of 3 time intervals. For the participants who completed 6 time intervals, a repeated measures ANOVA with the within-subjects factor 'time' (levels: before time interval 1, after time interval 2, before time interval 3 , after time interval 4 , before time interval 5 , after time interval 6 ) was calculated.

In the repeated measures ANOVAs, when the sphericity assumption was not met, the degrees of freedom and thus the $p$-values were corrected according to the Huynh-Feldt procedure. All post-hoc analyses were conducted by means of Duncan's Multiple Range tests. 


\section{Results}

Regarding the disengagement costs, the repeated measures ANOVA yielded a significant main effect of the factor 'time interval' $\left(F_{2,58}=7.98, p=.001\right)$, but no significant main effect of the factor 'saccade direction' $\left(F_{1,29}=3.65, p=.066\right)$. Overall, disengagement costs increased between the first and second time interval, but not between the second and third time interval. More relevantly, there was also asignificant interaction between the factors('time interval*saccade direction': $F_{2,58}=5.37 ; p=$ .007).The results concerning this interaction and the corresponding post-hoc tests are depicted in Fig. 3. For time intervals 1 and 2, there were no significant differences between disengagement costs for left- and rightward saccades. However, disengagement costs of the third time interval were significantly lower for rightward saccades than forleftward saccades. The repeated measures ANOVA for the 6 time intervals revealed similar results ('time interval*saccade direction': $F_{5,45}=2.58, p=$ .039, 'time interval': $F_{5,45}=1.2, p=.33$; 'saccade direction': $F_{1,9}=3.53, p=.093$ ). Post-hoc analyses revealed a continuationover time intervals 4,5 , and 6 of the significant difference between the disengagement costs for left- and rightward saccades, with lower costs for rightward saccades(see Fig.3).

Fig. 3 about here

To further investigate the origin of the effects ofincreasing time-on-task on disengagement costs, separate analyses were conducted on saccade latenciesfor the two tasks (gap, overlap) and the two saccade directions (left, right). The repeated measures ANOVA for the first 3 time intervals revealed no significant effect of the factor 'saccade direction' $\left(F_{1,29}=.84, p=.37\right)$ and no significant interactions 
between the factors 'time interval*saccade direction' $\left(\mathrm{F}_{2,58}=.69, p=.51\right)$ or 'saccade direction*task' $\left(F_{1,29}=.65, p=.07\right)$. However, there were significant main effects of the factors 'time interval' $\left(F_{2,58}=\right.$ 3.44; $p=.039)$ and 'task' $\left(F_{1,29}=211.34, p<.001\right)$. Regarding the former, saccadic latency decreased during time intervals 2 and $3(M=161.67, S E M=5.03 ; M=163.27, S E M=5.19)$ compared to time interval $1(M=168.43, S E M=5.56)$. Regarding the significant main effect of task, mean saccade latency was significantly lower for gap compared to overlap trials, thus reflecting the gap effect. Furthermore, there was a significant interaction between the factors 'time interval ${ }^{*}$ task' $\left(F_{2,58}=7.98\right.$, $p=.001$ ), reflecting a decrease in mean saccade latency for gap trials only during time intervals 2 and 3 compared to time interval 1 . Finally, and more relevantly, the analysis revealed a significant interaction between the factors 'time interval*saccade direction*task' $\left(F_{2,58}=5.37, p=.007\right)$. The results concerning this interaction and the corresponding post-hoc tests are listed in Tab. 1. For the gap task, there were no significant lateral differences regarding mean saccade latencyfor the first three time intervals. For the overlap task, no lateral differences were found in the first or the second time interval. However, in the third time interval, mean saccade latency was significantly lower for rightward than for leftward saccades. The repeated measures ANOVA for the 6 time intervals revealed similar results ('task': $F_{1,9}=51.1, p<.001$; 'time interval*saccade direction*task': $F_{5,45}=2.58$, $p=.039 ;$ 'time interval': $F_{5,45}=1.64, p=.17 ;$ 'saccade direction': $F_{1,9}=.77, p=.43$; 'time interval*saccade direction': $\mathrm{F}_{5,45}=.46, p=.81$; 'bock*task': $\mathrm{F}_{5,45}=1.2, p=.33$; 'saccade direction*task': $\left.\mathrm{F}_{1,9}=3.53, p=.093\right)$. Post-hoc tests for the 6 time intervals revealed a continuation of the significant difference in latency between leftward and rightward saccades for the overlap task during time intervals 4, 5, and 6.However, there were no constant significant differences for the gap task during time intervals 4, 5, and 6 (see Tab. 1), as asignificant lateral difference was observed fortime interval 4, but not for time intervals 5 and 6 . The presence of a single significant lateral difference in the gap task during time interval 4 is difficult to interpret, and might reflect aphysiological variability fluctuation. 
For peak saccadic velocity, the repeated measures ANOVA for the first 3 time intervals revealed a significant effect of the factor 'time interval' $\left(F_{2,58}=6.42, p=.003\right)$, but not of the factors 'saccade direction' $\left(\mathrm{F}_{1,29}=2.58, p=.12\right)$ or 'task' $\left(\mathrm{F}_{1,29}=1.23, p=.28\right)$, nor of the interactions 'time interval*saccade direction' $\left(F_{1.11,32.16}=.69, p=.43\right)$, 'time interval*task' $\left(F_{2,58}=.094, p=.91\right)$, 'saccade direction*task' $\left(F_{1,29}=.037, p=.85\right)$, or 'time interval*saccade direction*task' $\left(F_{2,58}=.053, p=.95\right)$. Irrespective of task and saccade direction, peak saccadic velocity decreased over the course of the 3 time intervals. Analysis of mean peak velocity for the 6 time intervals demonstrated no significant differences in mean peak velocity between time intervals 3 and time intervals 4,5 and 6 , respectively(see Fig. 4).

To exclude a possible decrease in velocity due to saccadichypometry, we next investigated whether saccade accuracy (gain) varied during the first 3 time intervals. The repeated measures ANOVA yielded a significant main effect of the factor 'task' $\left(F_{1,29}=4.41, p=.044\right)$. Saccade accuracy was significantly higher (i.e., gain values closer to 1 ) for overlap compared to gap trials (overlap: $M=.95$, SEM $=.01$; gap: $M=.94$, SEM $=.01)$. However, none of the other factors or interactions were significant ('time interval': $F_{2,58}=.5, p=.6$; 'saccade direction': $F_{1,29}=.22, p=.64$; 'time interval*saccade direction': $F_{1.56,45.2}=.61, p=.51$; 'time interval*task': $F_{2,58}=.056, p=.95$; 'saccade direction*task': $F_{1,29}=4, p=.055$; 'time interval*saccade direction*task': $\left.F_{2,58}=.42, p=.66\right)$. Hence, participants' saccade accuracy did not vary during the course of the experiment, nor was there any 
difference between left- and rightward saccades. Similar results were found for the 6 time intervals ('task': $F_{1,9}=9.62, p=.013$; 'time interval': $F_{2.3,20.7}=1.83, p=.18$; 'saccade direction': $F_{1,9}=3.05, p=.12$; 'time interval*saccade direction': $F_{1.64,14.77}=1.28, p=.3$; 'time interval*task': $F_{5,45}=.36, p=.87$; 'saccade direction*task': $F_{1,9}=3.65, p=.088$; 'time interval*saccade direction*task': $F_{5,45}=1.64, p=$ $.17)$.

Regarding the VAS of the 20 participants for whom the experiment ended after the completion of the 3 time intervals, the repeated measures ANOVA revealed a significant main effect of the factor 'time' $\left(F_{3,57}=13.86 ; p<.001\right)$. Post-hoc comparisons yielded a significant increase in subjective fatigue when comparing the VAS-scores obtained before time interval 1 with the ones obtained after time interval 2. Moreover, subjective fatigue decreased during the break between time intervals 2 and 3 , and increased again during time interval 3. For the participants who completed 6 time intervals, the repeated measures ANOVA also yielded a significant main effect of the factor 'time' $\left(F_{5,45}=18.93 ; p<\right.$ .001). Participants' fatigue decreased significantly during the break between time intervals 4 and 5 and increased significantly during task performance during time intervals 5 and 6 (see Fig. 5).

Fig. 5 about here 


\section{Discussion}

In the present study, we investigated whether prolonged time-on-task would result in differential disengagement costs for left- and rightward targets, as measured in a saccadic gap/overlap task. With increasing time-on-task, we found lower disengagement costs for rightward- compared to leftward saccades. These differences appeared from the third time interval of the task (i.e., after approx. 16 minutes of task performance), and were preserved during the ensuing time intervals (i.e., during approx. 32 minutes of task performance). The lateralized effects of increasing time-on-task on disengagement costs were primarily attributable to concurringside differences of saccadic latencies in overlap trials, and not in gap trials. As an additional finding, participants demonstrated decreasing saccadic peak velocities, which could not be attributed to changes in saccadic metrics. Finally, on a subjective level, participants reported a significant increase in fatigue during task performance (i.e., between the beginning and the end of the time intervals of the task), and a decrease during the short breaks between time intervals.

Disengagement costs (i.e., the differences in latencies between gap and overlap) have been shown to reflect the process of disengagement of attention (Fischer \&Breitmeyer, 1987; Fischer \& Weber, 1993; Jin\& Reeves, 2009). Our findings show that, in this context, the effects of fatigue due to increased time-on-task: a) are specific to the disengagement of attention, i.e., are mainly observable in overlap trials (in which attention has to be disengaged from central fixation) and not in gap trials (in which disengagement is not or less necessary due to central fixation extinction); and, b) influence the disengagement of attention asymmetrically, depending on the direction towards which attention needs to be displaced(i.e., disengagement costs become lower for right- than for leftward saccades). These results support the idea that non-spatial attentional aspects can have a significant influence on the deployment of attention in space. Previous studies in healthy controls manipulated non-spatial attentional aspects, e.g., increased fatigue by means of prolonging time-on-task, or reduced participants' arousal level (which refers to a more general, sleep-wake regulated activation of the 
cerebral cortex; Oken, Salinsky, \&Elsas, 2006) by means of sleep deprivation. As spatial attentional tasks, they then applied the landmark task (i.e., judging the accuracy of the bisection point in prebisected lines), or different detection tasks, in which lateral targets had to be detected covertly (i.e., keeping gaze at central fixation, without eye movements). Overall, these studies found a shift from a leftward to a rightward attentional bias, which was associated with the aforementioned non-spatial attentional manipulations (e.g., Benwell et al., 2013; Fimm, Willmes, \&Spijkers, 2006; Herber, Valvoda, Kuhlen, \&Fimm, 2008; Manly et al., 2005; Newman et al., 2013).The present study used a saccadic paradigm, in which attention had to be laterally displaced in an overt fashion (i.e., by means of eye movements), to investigate the impact of fatigue due to prolonged time-on-task. The advantage of this approach is that, on the one hand, the overt deployment of attention to the lateral targets can be precisely measured and, on the other hand, the disengagement of attention can be explicitly quantified (i.e., by means of the comparison between gap and overlap trials).

Possible left-right asymmetries in saccadic latencies have been assessed in earlier eye movement studies (e.g., Beydagi, Yilmaz, \&Suer, 1999; De Clerck, Crevits, \& van Maele, 2000; Honda, 2002; Vergilino-Perez et al., 2012). However, in these studies, the effects of fatigue due to increasing timeon-task were not specifically assessed, and much smaller numbers of trials were administered (i.e.,below 240 trials). The aforementioned studies, on a group level, did not find lateral differences in saccadic latencies for gap or for overlap trials during short time-on-task periods. This null finding for short time intervals is in line with the results of the present study, since, in our experiment, left/right asymmetries were observable only after longer time-on-task (i.e., after 240 trials, remaining up to 720 trials).This suggests that, in healthy participants,administering tasks for a longer time period is necessary in order to trigger measurable, lateralized effects of fatigue on disengagement costs, depending on the direction of the subsequent attentional deployment.In the second half of the task,we also observed an increase in the variance of saccade latencies for the overlap compared to the gap task. An increase in variance in behavioural performance in attentional tasks has previously been shown by Benwell et al. (2013). Overlap trials, which comprise attentional 
disengagement processes, require more extensive attentional processing than gap trials, which encompass no or less attentional disengagement. We speculate that the greater increase in variance over time-on-task for the overlap compared to the gap condition reflects the differences in attentional processing for these two conditions.

The influence of non-spatial attentional aspects (such as fatigue) on spatial attentional deployment can be tentatively explained within the framework of the right-hemispheric dominant representation of attention in the human brain (e.g., Thiebaut de Schotten et al., 2011), in particular for non-spatial attentional aspects (see, e.g., Corbetta\& Shulman, 2002).For instance,previous research has shown that patients with hemispatial neglect after right-hemispheric lesions show a decreased level of alertness, which refers to a general response readiness to sensory stimuli (e.g. Sturm \&Willmes, 2001), and deficits in sustaining attention, i.e., voluntarily maintaining attention over a prolonged period of time (Samuelsson, Hjelmquist, Jensen, Ekholm, \&Blomstrand, 1998; Sturm \&Willmes, 2001; see alsoLangner\&Eickhoff, 2014).Moreover, their performance in a sustained attention task has been shown to be apredictor ofspatial attentional performance (Robertson et al., 1997).In line with these findings, a recent meta-analysis of data obtained in healthy controls (Langner\&Eickhoff, 2014) evidenceda predominantly right-hemisphericcortico-subcortical network involved in the maintenance of vigilance,a form of sustained attention.This right-hemispheric dominancefor nonspatial attentional aspects isthought to be due to asymmetrical projectionsfrom the noradrenergic locus coeruleus, which is involved in arousal and vigilance regulation (Corbetta, Patel, \& Shulman, 2008;Posner \& Petersen 1990).Overall, a reduction in non-spatial attentional performance would thus affect activity more prominently in the right than the left hemisphere. Assuming that interhemispheric rivalry dynamics direct spatial attentional deployment (e.g., Kinsbourne, 1993; Corbetta\&Shulman, 2011), this would thus result in the observed rightward shift in spatial attentional deployment with increasing time-on-task and fatigue,and/or with a decreasing level of arousal. This prediction is in line with the results of the present study, and with the rightward bias in spatial attentional deployment with increasing time-on-task and/or a decreasing arousal level 
observed in previous studies (e.g., Benwell et al., 2013; Fimm et al., 2006; Herber et al., 2008; Manly et al., 2005; Newman et al., 2013).

Interestingly, the results of the present study show that the lateral differences in disengagement costs can be primarily attributed to a decrease in saccadic latencies for rightward saccades in the overlap trials, rather than to an increase for leftward saccades. Hence, the results can be primarily interpreted as a facilitation of disengagement for rightward saccades, rather than an increase of disengagement costs for leftward saccades.A facilitation of rightward attentional shiftsis sometimes observed in patients suffering from left-sided hemispatial neglect, a phenomenonoften referred to as "magnetic attraction" towards the right, ipsilesional visual space. In fact, neglect patients can exhibit an ipsilesional saccadic facilitation, which results in inappropriate ipsilesional saccades or markedly quicker ipsilesional saccadic reaction times (e.g., Bourgeois et al., 2015; Natale, Marzi, Bricolo, Johannsen, \&Karnath, 2007). In an overlap saccadic task, whilst having contralesional difficulties in saccadic stimulus detection, neglect patients could even outperform healthy subjects with their shortened rightward saccadic reaction times (Natale et al., 2007).

Another important aspect of the findings of the present study is that lateralized effects of increasing time-on-task were specific to attentional disengagement, i.e., were robust only for the overlap task and not for the gap task. A possible explanation is that the extinction of the central fixation in the gap trials can act as an alerting signal, preparing participants for the forthcoming appearance of a target stimulus, a process which is absent in overlap trials (Reuter-Lorenz, Oonk, Barnes, \& Hughes, 1995). One can postulate that the presence of an alerting signal can counteract, at least short-term, the negative effects of fatigue due to increasing time-on-task. In keeping with the aforementioned interactions between spatial and non-spatial attentional aspects, lower fatigue would, in turn, trigger a spatial attentional deployment that is more balanced over both sides of space, and thus no lateralized effects in gap trials would occur. Following this tentative explanation, previous findings show that the detection of contralesional stimuli in neglect patients improves for a short time period 
after the presentation of an alerting tone (Chica et al., 2012; Robertson, Mattingley, Rorden, \& Driver, 1998; van Vleet\& Robertson, 2006).

On an objective level, our results indicate that participants did not benefit from the short breaks between the time intervals of the task. However, on a subjective level, participants indicated a decrease of fatigue after the breaks. The fact that the level of fatigue in the participants did increase, irrespectively of the short breaks, is supported by the observed saccadic velocity decrease. Participants showed a decrease in saccadic velocity over the course of the first 3 time intervals, which then did not decrease any further, but remained low for the following time intervals 4 to 6 , possibly reflecting a floor effect. Saccadic velocity has been shown to be a reliable indicator of mental fatigue due to prolonged time-on-task in several previous studies (e.g., Cazzoli et al., 2014; Di Stasi, Catena, Cañas, Macknik, \& Martinez-Conde, 2013; Di Stasi et al., 2012). A mismatch between subjective and objective measurements of fatigue is a common finding (Christodoulou, 2005), even regarding the effect of a short break (subjectively effective, but objectively ineffective) after prolonged time-on-task (Di Stasi et al., 2012).Regarding the visual analogue scale, an attentional bias with increasing time on-task might also bias participants' perception of the horizontal scale (analogously to rightward shifts in landmark tasks; e.g., Manly et al., 2005) .As fatigue decreased from left to right in the visual analogue scale of the present study, a rightward attentional bias might have corresponded to a slightly biased decreasein subjective fatigue. Hence, in order to avoid a possible impact of an attentional bias, a vertical scale or a horizontal scale with maximum fatigue at the left- /rightward extreme for $50 \%$ of the trials might be a promising alternative approach for future studies.

In conclusion, our results demonstrated lateral differences regarding the speed of attentional disengagement with fatigue due to increasing time-on-task using a saccadic gap/overlap paradigm. In particular, with increasing time-on-task, our participants demonstrated lower disengagement costs for rightward compared to leftward saccades. The results support the notion of an interaction between non-spatial and spatial attentional aspects, whereby non-spatial attentional aspects can 
substantially influence the disengagement of spatial attention, depending on the direction of its subsequent deployment. 


\section{Acknowledgements}

This study was supported by the Swiss National Science Foundation (Ambizione Grant No. PZ0OP3_154714/1). 


\section{References}

Benwell, C. S. Y., Harvey, M., Gardner, S., \&Thut, G. (2013). Stimulus- and state-dependence of systematic bias in spatial attention: additive effects of stimulus-size and time-on-task. Cortex, 49(3), 827-836. doi:10.1016/j.cortex.2011.12.007

Beydagi, H., Yilmaz, A., \& Suer, C. (1999). The effect of direction on saccadic eye movement parameters.Journal of Basic and Clinical Physiology and Pharmacology, 10(1), 73-77.

Bocca, M.-L., \& Denise, P. (2006). Total sleep deprivation effect on disengagement of spatial attention as assessed by saccadic eye movements. Clinical Neurophysiology, 117(4), 894-899. doi:10.1016/j.clinph.2006.01.003

Bourgeois, A., Chica, A. B., Migliaccio, R., Bayle, D. J., Duret, C., Pradat-Diehl, P., . . Bartolomeo, P. (2015). Inappropriate rightward saccades after right hemisphere damage: oculomotor analysis and anatomical correlates. Neuropsychologia, 73, 1-11. doi:10.1016/j.neuropsychologia.2015.04.013

Cazzoli, D., Antoniades, C. A., Kennard, C., Nyffeler, T., Bassetti, C. L., \& Müri, R. M. (2014). Eye movements discriminate fatigue due to chronotypical factors and time spent on task-a double dissociation. PLoSONE, 9(1), e87146. doi:10.1371/journal.pone.0087146

Chica, A. B., Thiebaut de Schotten, M., Toba, M., Malhotra, P., Lupiáñez, J., \& Bartolomeo, P. (2012). Attention networks and their interactions after right-hemisphere damage. Cortex, 48(6), 654-663. doi:10.1016/j.cortex.2011.01.009

Christodoulou, C. (2005) The assessment and measurement of fatigue. In J. DeLuca (Ed.), Fatigue as a window to the brain (19-35). Cambridge: MIT Press.

Corbetta, M., \& Shulman, G. L. (2002).Control of goal-directed and stimulus-driven attention in the brain.Nature reviews Neuroscience, 3(3), 201-215. doi:10.1038/nrn755

Corbetta, M., \& Shulman, G. L. (2011).Spatial neglect and attention networks.Annual Review of Neuroscience, 34, 569-599. doi:10.1146/annurev-neuro-061010-113731 
Corbetta, M., Akbudak, E., Conturo, T. E., Snyder, A. Z., Ollinger, J. M., Drury, H. A., . . Shulman, G. L. (1998). A common network of functional areas for attention and eye movements.Neuron, 21(4), 761-773. doi:10.1016/S0896-6273(00)80593-0

Corbetta, M., Patel, G., \& Shulman, G. L. (2008). The reorienting system of the human brain: from environment to theory of mind. Neuron, 58(3), 306-324. doi:10.1016/j.neuron.2008.04.017

De Clerck, M., Crevits, L., \& van Maele, G. (2000). Saccades: is there a difference between right and left? Neuro-Ophthalmology, 24(2), 327-330. doi:10.1076/noph.24.2.327.7157

Di Stasi, L. L., Catena, A., Cañas, J. J., Macknik, S. L., \& Martinez-Conde, S. (2013). Saccadic velocity as an arousal index in naturalistic tasks. Neuroscience and Biobehavioral Reviews, 37(5), 968-975. doi:10.1016/j.neubiorev.2013.03.011

Di Stasi, L. L., Renner, R., Catena, A., Cañas, J. J., Velichkovsky, B. M., \&Pannasch, S. (2012). Towards a driver fatigue test based on the saccadic main sequence: a partial validation by subjective report data.Transportation Research Part C: Emerging Technologies, 21(1), 122-133. doi:10.1016/j.trc.2011.07.002

Dufour, A., Touzalin, P., \& Candas, V. (2007). Time-on-task effect in pseudoneglect.Experimental Brain Research, 176(3), 532-537. doi:10.1007/s00221-006-0810-2

Fimm, B., Willmes, K., \&Spijkers, W. (2006).The effect of low arousal on visuo-spatial attention.Neuropsychologia, 44(8), 1261-1268. doi:10.1016/j.neuropsychologia.2006.01.027

Fischer, B., \&Breitmeyer, B. (1987).Mechanisms of visual attention revealed by saccadic eye movements.Neuropsychologia, 25(1A), 73-83.

Fischer, B., \& Weber, H. (1993). Express saccades and visual attention. Behavioral and Brain Sciences, 16(3), 553-610. doi:10.1017/S0140525X00031575

Grandjean, E. (1979). Fatigue in industry.Occupational and Environmental Medicine, 36(3), 175-186. doi:10.1136/oem.36.3.175 
Heber, I. A., Valvoda, J. T., Kuhlen, T., \& Fimm, B. (2008). Low arousal modulates visuospatial attention in three-dimensional virtual space. Journal of the International Neuropsychological Society, 14(2), 309-317. doi:10.1017/S135561770808034X

Hoffman, J. E., \&Subramaniam, B. (1995).The role of visual attention in saccadic eye movements.Perception \& Psychophysics, 57(6), 787-795. doi:10.3758/BF03206794

Honda, H. (2002). Idiosyncratic left-right asymmetries of saccadic latencies: examination in a gap paradigm. Vision Research, 42(11), 1437-1445. doi:10.1016/S0042-6989(02)00050-0

Jin, Z., \& Reeves, A. (2009).Attentional release in the saccadic gap effect.Vision Research, 49(16), 2045-2055. doi:10.1016/j.visres.2009.02.015

Kingstone, A., \& Klein, R. M. (1993). What are human express saccades? Perception \& Psychophysics, 54(2), 260-273. doi:10.3758/BF03211762

Kinsbourne, M. (1993).Orientational bias model of unilateral neglect: Evidence from attentional gradients within hemispace. In I. H. Robertson \& J. C. Marshall (Eds.), Unilateral neglect: Clinical and experimental studies. Brain damage, behaviour \& cognition series, (pp. 63-86). Hillsdale, NJ, England: Lawrence Erlbaum Associates.

Lal, S. K., \& Craig, A. (2001).A critical review of the psychophysiology of driver fatigue.Biological Psychology, 55(3), 173-194. doi:10.1016/\$0301-0511(00)00085-5

Langner, R., \&Eickhoff, S. B. (2013).Sustaining attention to simple tasks: a meta-analytic review of the neural mechanisms of vigilant attention.Psychological Bulletin, 139(4), 870-900. doi:10.1037/a0030694

Manly, T., Dobler, V. B., Dodds, C. M., \& George, M. A. (2005).Rightward shift in spatial awareness with declining alertness.Neuropsychologia, 43(12), 1721-1728. doi:10.1016/j.neuropsychologia.2005.02.009 
Natale, E., Marzi, C. A., Bricolo, E., Johannsen, L., \&Karnath, H.-O. (2007). Abnormally speeded saccades to ipsilesional targets in patients with spatial neglect. Neuropsychologia, 45(2), 263-272. doi:10.1016/j.neuropsychologia.2006.07.008

Newman, D. P., O'Connell, R. G., \&Bellgrove, M. A. (2013). Linking time-on-task, spatial bias and hemispheric activation asymmetry: a neural correlate of rightward attention drift. Neuropsychologia, 51(7), 1215-1223. doi:10.1016/j.neuropsychologia.2013.03.027

Nobre, A. C., Gitelman, D. R., Dias, E. C., \&Mesulam, M. M. (2000). Covert visual spatial orienting and saccades: overlapping neural systems. Neurolmage, 11(3), 210-216. doi:10.1006/nimg.2000.0539

Pereverzev, V. (2014).Fatigue and mental performance during prolonged mental activities on fasting in young people with different attitudes to alcohol use.Journal of Behavioral Health, 3(3), 192199. doi:10.5455/jbh.20140811101711

Oken, B. S., Salinsky, M. C., \&Elsas, S. M. (2006). Vigilance, alertness, or sustained attention: physiological basis and measurement. Clinical Neurophysiology, 117(9), 1885-1901. doi:10.1016/j.clinph.2006.01.017

Oldfield, R. C. (1971). The assessment and analysis of handedness: The Edinburgh inventory. Neuropsychologia, 9(1), 97-113. doi:10.1016/0028-3932(71)90067-4

Posner, M. I., \& Petersen, S. E. (1990).The attention system of the human brain.Annual Review of Neuroscience, 13, 25-42. doi:10.1146/annurev.ne.13.030190.000325

Posner, M. I., Cohen, Y., \&Rafal, R. D. (1982).Neural systems control of spatial orienting. Philosophical Transactions of the Royal Society B: Biological Sciences, 298(1089), 187-198. doi:10.1098/rstb.1982.0081

Reuter-Lorenz, P. A., Oonk, H. M., Barnes, L. L., \& Hughes, H. C. (1995). Effects of warning signals and fixation point offsets on the latencies of pro- versus antisaccades: Implications for an interpretation of the gap effect. Experimental Brain Research, 103(2), 28793.doi:10.1007/BF00231715 
Robertson, I. H., Manly, T., Beschin, N., Daini, R., Haeske-Dewick, H., Hömberg, V.,. . . Weber, E. (1997). Auditory sustained attention is a marker of unilateral spatial neglect. Neuropsychologia, 35(12), 1527-1532. doi:10.1016/S0028-3932(97)00084-5

Robertson, I. H., Mattingley, J. B., Rorden, C., \& Driver, J. (1998). Phasic alerting of neglect patients overcomes their spatial deficit in visual awareness. Nature, 395(6698), 169-172. doi:10.1038/25993

Samuelsson, H., Hjelmquist, E. K., Jensen, C., Ekholm, S., \&Blomstrand, C. (1998). Nonlateralized attentional deficits: an important component behind persisting visuospatial neglect? Journal of Clinical and Experimental Neuropsychology, 20(1), 73-88. doi:10.1076/jcen.20.1.73.1481

Saslow, M. G. (1967). Effects of components of displacement-step stimuli upon latency for saccadic eye movement.Journal of the Optical Society of America, 57(8), 1024-1029. doi:10.1364/JOSA.57.001024

Shepherd, M., Findlay, J. M., \& Hockey, R. J. (1986). The relationship between eye movements and spatial attention. The Quarterly Journal of Experimental Psychology Section A, 38(3), 475-491. doi:10.1080/14640748608401609

Sturm, W., \&Willmes, K. (2001).On the functional neuroanatomy of intrinsic and phasic alertness.Neurolmage, 14(1 Pt 2), S76-84. doi:10.1006/nimg.2001.0839

Thiebaut de Schotten, M., Dell'Acqua, F., Forkel, S. J., Simmons, A., Vergani, F., Murphy, D. G. M., \& Catani, M. (2011).A lateralized brain network for visuospatial attention.Nature Neuroscience, 14(10), 1245-1246. doi:10.1038/nn.2905

vanVleet, T. M., \& Robertson, L. C. (2006). Cross-modal interactions in time and space: auditory influence on visual attention in hemispatial neglect. Journal of Cognitive Neuroscience, 18(8), 1368-1379. doi:10.1162/jocn.2006.18.8.1368

Vergilino-Perez, D., Fayel, A., Lemoine, C., Senot, P., Vergne, J., \& Doré-Mazars, K. (2012). Are there any left-right asymmetries in saccade parameters? Examination of latency, gain, and peak 
velocity.Investigative Ophthalmology \& Visual Science, 53(7), 3340-3348. doi:10.1167/iovs.119273 


\section{Figure Captions}

Fig. 1.Gap and overlap trials. Time course of a gap (top) and an overlap (bottom) trial.

Fig. 2.Procedure of the experiment. 30 participants completed 3 time intervals (TI), i.e., 360 trials. In addition, they filled in the visual analogue scale multiple times during the course of the experiment. 10 out of the 30 participants, who completed the first 3 time intervals, also completed further 3 time intervals.

Fig. 3. Mean disengagement costs. Mean disengagement costs for left- and rightward saccades, depicted separately for each time interval. Results of the first three time intervals were obtained in 30 subjects (left part of the figure), whereas the results for the time intervals 4 to 6 were obtained in the 10 subjects who completed 6 time intervals (right part of the figure). Error bars represent the standard error of the mean (SEM). Significant post-hoc tests are depicted by means of asterisks $\left({ }^{* *} p<\right.$ $.01 ; * p<.05)$.

Fig. 4. Mean peak saccadic velocity. Mean peak saccadic velocity for the first three time intervals were obtained in 30 subjects (left part of the figure), whereas the results for the time intervals 4 to 6 were obtained in 10 subjects who completed 6 time intervals (right part of the figure). Error bars represent the standard error of the mean (SEM).

Fig. 5. Mean scores of the visual analogue scale (VAS), reflecting the subjective level of fatigue. Higher scores indicate a higher level of fatigue. Black bars represent the scores obtained in the 20 participants who completed 3 time intervals. Grey bars represent the scores obtained in the 10 participants who completed 6 time intervals. Error bars represent the standard error of the mean (SEM). Significant post-hoc tests are depicted by means of asterisks $\left({ }^{* *} p<.01 ;{ }^{*} p<.05\right)$. 


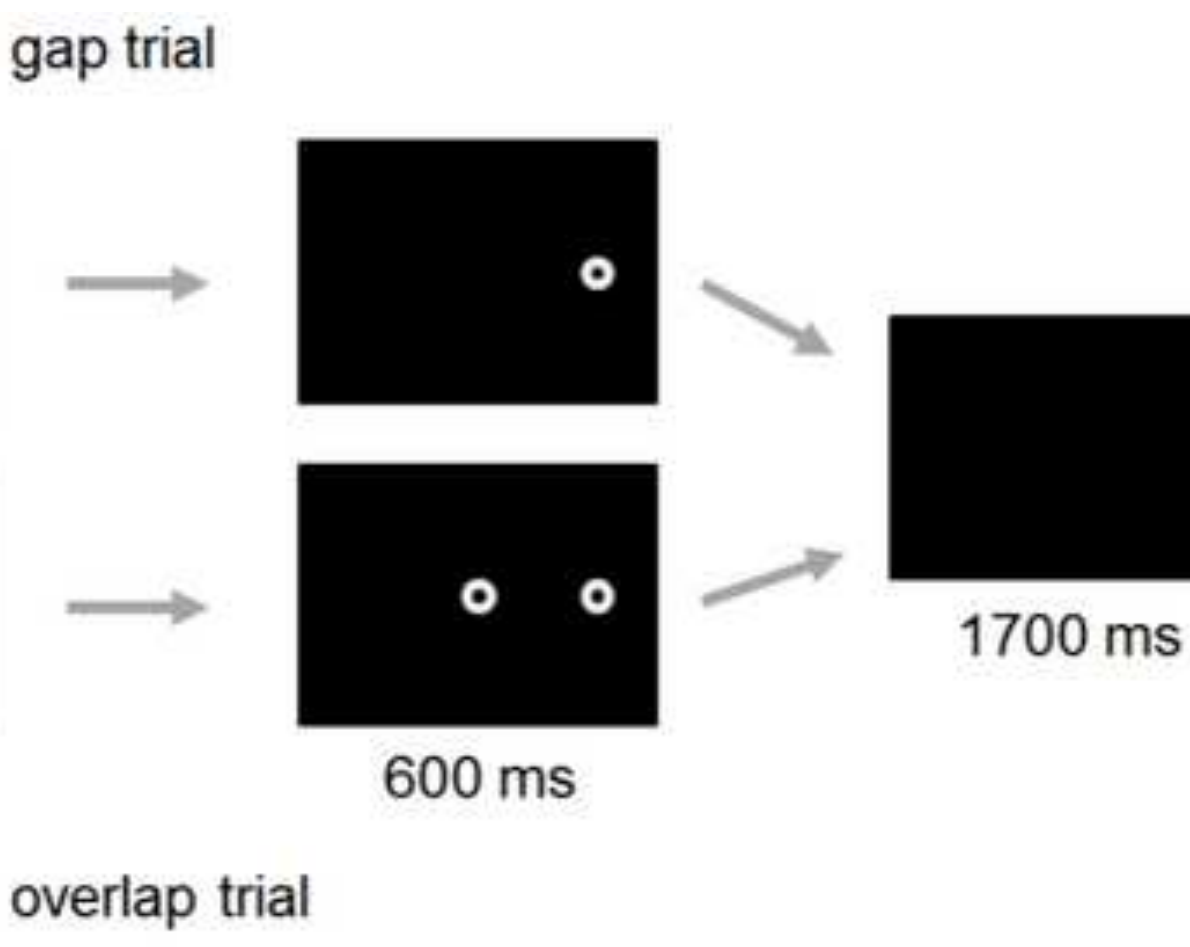

overlap trial

gap trial

\section{$600 \mathrm{~ms}$}

$1700 \mathrm{~ms}$

( 


$$
N=30
$$

\begin{tabular}{|c|c|c|c|}
\hline TI 1 & TI 2 & Bl & TI 3 \\
(120 trials $)$ & (120 trials $)$ & Break & (120 trials) \\
\hline
\end{tabular}

$N=10$ (out of the 30 )

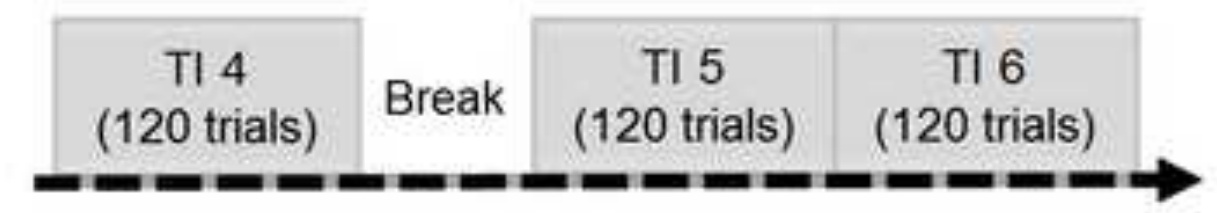




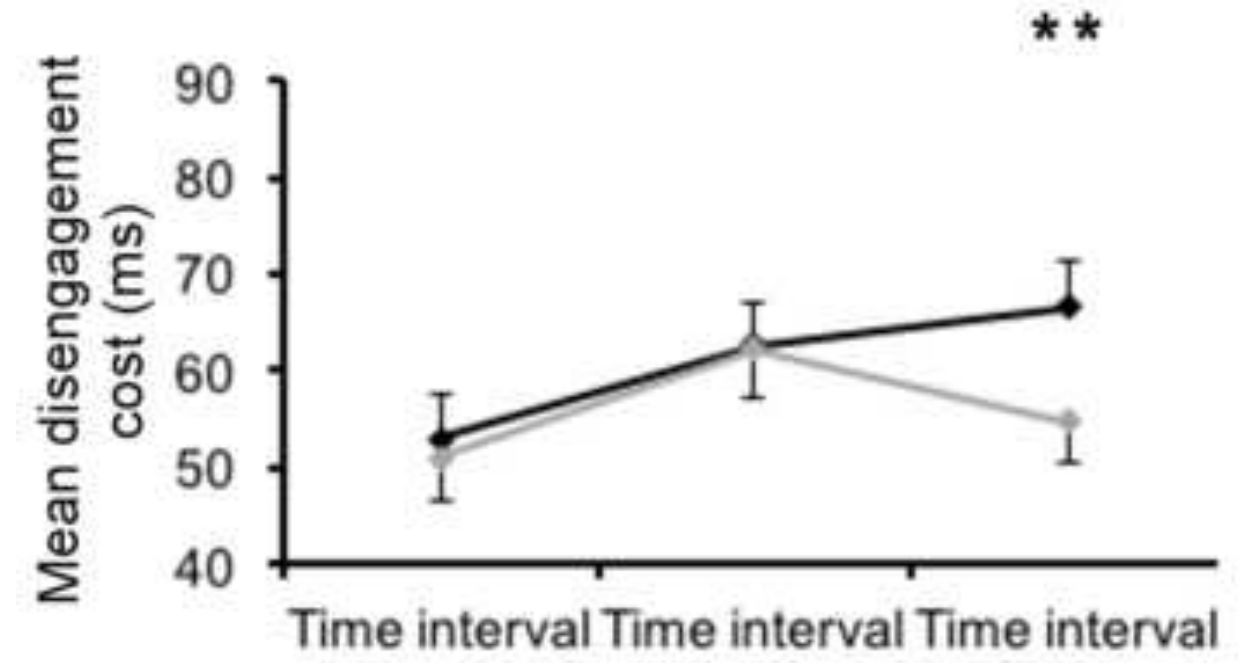

1
2
3
* *
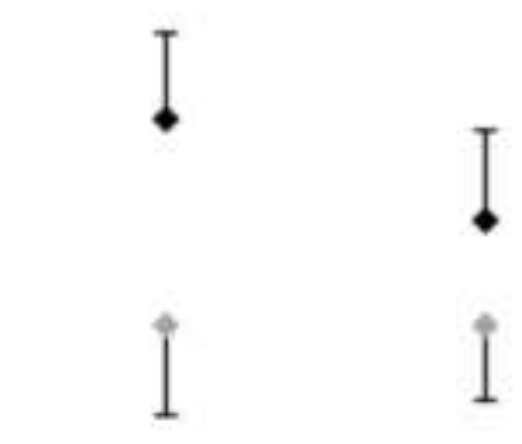

Ti

Time inter

4

*
*

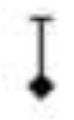

1

- left

$\rightarrow$ right 


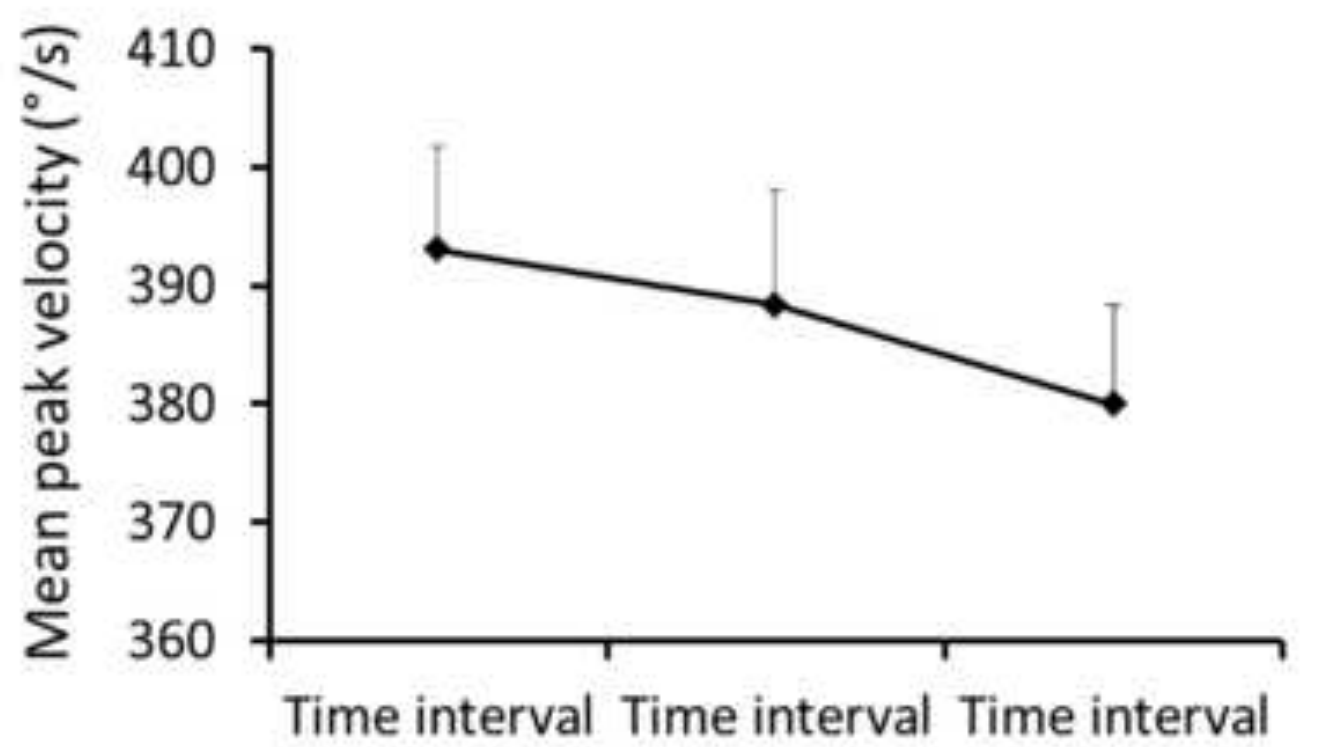

1
2
3
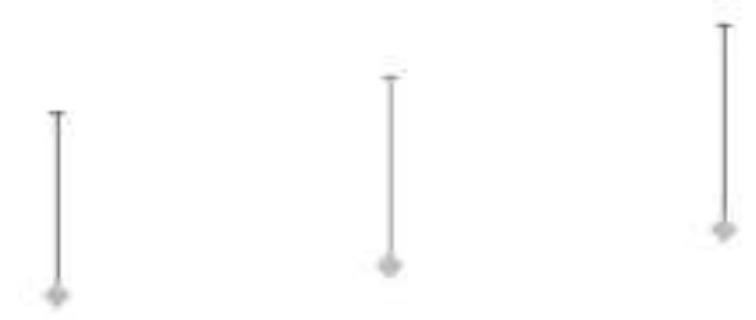

Time interval Time interval Time interval

4

5

6 

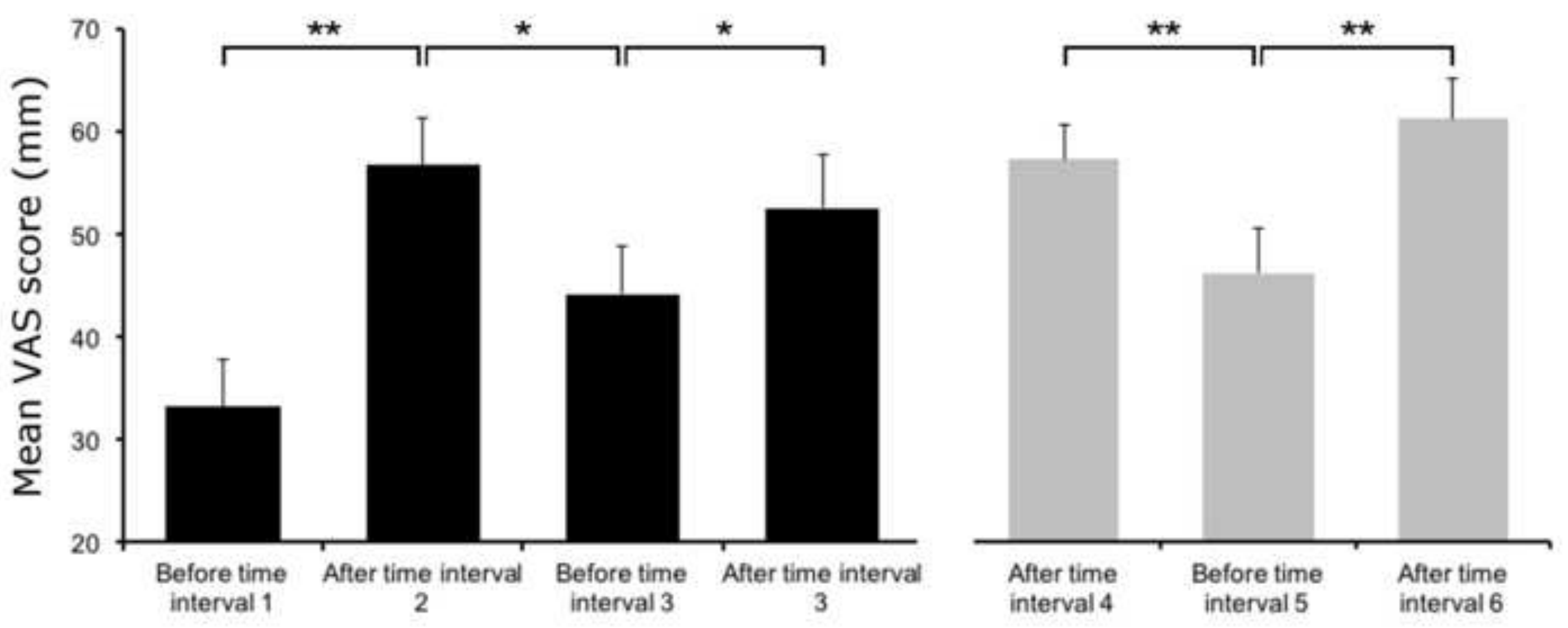